\title{
The Effect of Dietary Products with Estrogenic Properties on Breast Cancer in Women Over 50
}

\author{
Anisha Mehta ${ }^{1}$, Kevin Fink ${ }^{1 *}$ and Aubrey Frantz ${ }^{2 \#}$ \\ ${ }^{1}$ Highland Park High School, Dallas, TX, USA \\ ${ }^{2}$ University of North Texas, Dallas, TX, USA \\ "Teacher, "Advisor
}

\section{$\underline{\text { ABSTRACT }}$}

There has been a growing number of cases of women with breast cancer over age 50. My test question is to what extent do dietary products with estrogenic properties affect the development of breast cancer in women over the age of 50 in the United States? Therefore, from this research question, my hypothesis is that breast cancer risk and diets that increase estrogen levels are correlated strongly with one another for women over age 50. I utilized correlational research to analyze if foods that women eat on a daily basis correlate with their levels of estradiol in one data set and thus analyze if these increased estradiol levels affect breast cancer risk. In order to do this, I looked at pre-existing data from a variety of medical journals. Through my results, it seemed that as estrogen dietary pattern score increased, the breast cancer risk increased. It was also seen that a western diet (red meat, high estrogenic diets) can increase breast cancer risk significantly and that a prudent diet (vegetables, fruits) does not affect the risk. During this research study, the most important limitation is that only studies from published medical journals were used. Some studies were not published online meaning the results remained private. Overall, my results of this study suggested that breast cancer risk and diets that increase estrogen levels are in fact correlated strongly with one another for women over age 50. The results of this study inspire further inquiry into medical applications of diets with estrogenic properties. Additionally, more studies can show how other hormones are correlated with breast cancer risk, and if so which kind of foods. Also, some future actions include motivational counseling techniques and education about healthy lifestyle choices.

\section{Introduction}

A high frequency of breast cancer in the population is one of many health concerns in American women over the age of 50 (1). In the month of January in 2020, the American Cancer Society reported that approximately $39.5 \%$ of women will be diagnosed with cancer at some point during their lifetimes (1). As statistically reported in a case study, almost one-quarter of all cancer deaths are due to lung cancer, more than breast, prostate, and colorectal cancers combined (2). Despite these cancers being different, the most common factors/carcinogens (toxic substances that can cause cancer) that can lead to the development of these cancers, specifically breast cancer are the same and include: smoking, an unhealthy diet, and even involuntary factors such as genetics (3). Malignant cancer (a type of cancer where the cells are cancerous and can spread to other tissues and organs) is the most detrimental type of cancer, while benign (where cancer cells remain intact in the cell) cancers are not as dangerous. According to the National Cancer Institute, one-quarter of these new malignant cancer cases are diagnosed in women aged 65 to 74 (2). A similar pattern is seen for many common cancer types. For example, the median age at diagnosis is 61 years for breast cancer, 68 years for colorectal cancer, 70 years for lung cancer, and 66 years for prostate cancer for women (2).

As the female baby boomers age, there has been a significant increase in many types of breast cancers in that age group (2), so researching what happens in female cancer patients over age 50 can control this cancer prevalence 
and be a major scientific breakthrough. Some reasons why cancer (specifically breast cancer) occurs in older age may be due to menopause, since a higher lifetime exposure to estrogen may increase breast cancer risk(4). Researchers have also shown how factors such as eating and having unhealthy habits can contribute to cancer and increase the mortality rate of patients (5). Some of these unhealthy habits include eating red meat, soy, and consuming sugary drinks. However, not many researchers have identified why women can still eat healthy, but still develop cancer. For example, not many researchers have not yet defined if eating various sources of fruits and vegetables is effective for preventing cancer, since many healthy women still are diagnosed with cancer. Some specific examples of this can be mirrored using mice (3).

According to Science Daily, the findings reveal how mice can mimic breast cancer tissue and its genes (3). In order to research more about this topic while considering ethics, this study can first use mice by giving them healthy and unhealthy products. The researcher can then measure the effectiveness of these dietary factors and the approximate decline of cancer risk in older female mice. This study begins with a discussion of the various types of cancer and investigates the root causes of these illnesses through the investigation of mice (6). The researcher was motivated to investigate the "influence of obesity on the entire process of breast carcinogenesis from the stages of early proliferation at the primary site to the formation of systemic metastases by feeding the mice a high-fat and high-calorie diet." (7) In this paper, the question addressed is how the development of malignant breast cancer can be prevented from dietary products with estrogenic properties in women over the age of 50 in the United States. This study's main focus aims to inform women over age 50 that there is a dramatic influence of dietary factors on breast cancer, and to identify why patients with healthy diets can still develop malignant cancer by estrogen. In 2006, researchers found that higher levels of Insulin-like Growth Factor 1 can increase the risk of breast cancer, and that higher amounts of hormones such as prolactin can also increase the risk of breast cancer (8). Other hormones such as estrogen are also linked to this because some breast cancer cells need estrogen to grow and when it attaches to special proteins called estrogen receptors, these cancer cells with the receptors grow (9). A study conducted in 2018 published in the International Journal of Cancer Research and Treatment, a journal publishing significant clinical studies from cancer programs around the world, found that since estrogen feeds and fuels 80 percent of all breast cancers via estrogen binding to an estrogen receptor on the cell's surface, the goal of some therapies is to reduce estrogen levels in the body and to keep it out of the receptors (like tamoxifen does) (10). In 2018, the author linked estrogen to the amount of menstrual cycles a woman goes through (11). For example, according to a group of researchers in the National Institute of Environmental Health Sciences, "Every month a woman's menstrual cycle stimulates breast tissue with estrogen that can attach to hormone receptors of breast cancer cells, so the less menstrual cycles you have, the lower your risk for ER positive breast cancer." (4). A woman's menstrual cycle is divided into 4 phases: the menstruation phase, follicular phase, ovulation phase, and luteal phase (10). In fact, breast cancer proliferation was found to be the most prevalent in the luteal phase then the follicular phase (11). The luteal phase is when the ruptured follicle closes after releasing the egg and forms a structure called a corpus luteum, which produces increasing quantities of progesterone (12). According to the American Association for Cancer Research (the world's oldest and largest professional association related to cancer research), due to these irregular menstrual cycles in some women, women who spend a smaller proportion of their menstrual cycle in the luteal phase may have a reduced risk of breast cancer (6). Therefore, as women age and have more menstrual cycles, the risk for breast cancer increases regardless if the patient is healthy. However, an article published from the American Association for Cancer Research Journals, "believes that increased age is regularly linked with heightened cancer risk, but recent research suggests a flattening around age 80 in women."(6). This article only discusses all types of cancers, and not just breast cancer which is dependent on estrogen levels. Even though women are not having menstrual cycles at age 80 , they still have a buildup of estrogen from before menopause. This buildup of estrogen often comes from what one eats as a study has concluded with mice (13).

In order to obtain nutrition to promote a healthy level of estrogen being released, dietary products have been studied and found to be either beneficial or detrimental for prevention of breast cancer. In fact, according to a clinical study published in the US National Library of Medicine in 2016, "nutrition influences cancer etiology in about 35\% 
of cancer cases." (5). Some of the products that many sources have proved to avoid include butter, processed meats, soy, and red clover, while products such as fruits and vegetables should be encouraged (9).

However, some articles have contrary findings stating that "recent large studies have not found that soy food intake affects breast cancer coming back or survival rates. While eating soy foods doesn't seem to pose a risk, the evidence regarding the effects of taking soy or isoflavone supplements is not as clear." (8). In fact, it has been concluded that "the safest way to consume soy if you're trying to prevent cancer (and have never had it before) is to eat unprocessed or fermented soy, such as edamame, tofu, or miso"(14). From the previous studies mentioned, it is clear that a woman's diet does have a great impact on the amount of estrogen their body releases, which can in turn have an effect on their risk for breast cancer. Therefore, my hypothesis is that breast cancer risk and diets that increase estrogen levels are correlated strongly with one another for women over age 50.

As stated previously, it has been identified that increased estrogen exposure as women age through menstrual cycles have a significant impact on breast cancer risk. Studies have also proven that breast cancer is related to the diet a woman adopts $(5,8,9)$. Therefore, the purpose of this paper is to find how estrogenic foods can cause breast cancer and to what extent this can happen. Many studies have published that items such as soy and foods with added sugar are detrimental (15), but not many have identified how the estrogen levels of these foods play a role in breast cancer risk.

\section{Results}

The first segment of the research was to explore more about what estrogen contains that causes breast cancer risk and what common foods contain estrogen on health websites that are credible and have a medical background. I needed to access specific health publishers like the National Cancer Institute, Susan G. Komen, National Library of Medicine, and International Journal of Cancer Research and Treatment, and even utilized Google Scholar which I accessed through my expert advisor. I then compiled all of my data in one google document and assessed which ones provided the most important results. After narrowing down the studies, I used the graphs to find a correlation between the two variables to see if the foods that the women eat on a daily basis correlate with their risk for breast cancer. I then looked through the studies that answered my research question a few weeks later after collecting data to determine if there is a link between elevated estrogen levels in women and their diet. To make sure the data is viable, I recorded the findings discussed in the meetings with my expert advisor to ensure that the correlation is accurate and precise. To be fit inside the research paper, the data for only women over age 50 was organized in tables for each research study (totaling up to 75 participants) which was included in my research paper. Since the data I utilized was online and no human subjects were involved, no face-to-face communication was required therefore increasing confidentiality. ${ }^{3 \mathrm{~S}}$

One study that tried to find a link between diets specifically Mediterranean types and breast cancer was published in the American Journal of Clinical Nutrition, a monthly peer-reviewed biomedical journal (14). The researchers cited in the journal found that "postmenopausal women who reported the highest intakes of dietary lignans were 14 percent less likely to develop breast cancer than those with low intakes." (17). The researchers concluded that the Mediterranean diet "cuts deadly breast cancer risk by $40 \%$ in postmenopausal women due to its lack of estrogen."(18). This means that estrogen in diet does play a role in a woman's risk for breast cancer.Another study that has narrowed their population to post-menopausal woman was the National Cancer Institute (13). The study listed their method stating that "computerized search of the English language literature on estrogen/estradiol and dietary fat intervention studies published from January 1966 through June 1998 was conducted using the MEDLINE database. Pooled estimates were derived from the change in estradiol levels associated with fat reduction from 13 studies. Analyses were conducted separately for premenopausal and postmenopausal women and in both groups combined (13). It was found that higher dietary fat consumption (Quartile 5) was correlated with higher estradiol levels and lower dietary fat consumption (Quartile 1) was correlated with lower estradiol levels.

The reason why women over the age of 50 were chosen specifically for my research was because in a Cancer Statistics Review in 2009 , the percent risk was $2.38 \%$ at age 50 and increased to $3.82 \%$ at age 60 (19).This is a dramatic increase 
since at age 30 , the percent risk was only $.44 \%$ at age 30 (19). Also, age 50 is the borderline age for menopause which serves a significant purpose when determining breast cancer risk (20). According to the University of Texas MD Anderson Cancer Center, it has been discovered that "if a woman goes through menopause after age 50, their risk for breast cancer is slightly higher than the other women who went through it from age 45-50" (21).

Table 1: Relative risk of breast cancer by estrogen dietary pattern score among women (22)

\begin{tabular}{|l|l|l|}
\hline Quartiles & Cases & Age-Adjusted \\
\hline Continuous & N/A & $1.03(1.01-1.05)$ \\
\hline Quartile 1 & 363 & 1.00 (reference) \\
\hline Quartile 2 & 401 & $1.10(0.92-1.30)$ \\
\hline Quartile 3 & 414 & $1.13(0.96-1.35)$ \\
\hline$p$-trend & N/A & $1.30(1.10-1.55)$ \\
\hline Quartile 4 & 425 & 0.003 \\
\hline
\end{tabular}

The table above shows quartile 1 as the people with a low estrogenic diet and quartile 4 as the people with a high estrogenic diet. The numbers in the table indicate the percent increase risk of breast cancer. For example, in this table, women in the highest quartile of estrogen pattern score (Quartile 4) had a 30\% (from 1.30) increased risk of breast cancer overall compared to women in the lowest quartile of estrogen pattern score (1.00) (22). Since the age-adjusted column has a p-trend lower than .05 , this shows that that column is reliable and statistically significant meaning that 
there is a significant difference between the different quartiles. Also, it is known that age is a factor of breast cancer, but this table shows that independent of age, the group with higher estrogen levels has a higher risk of breast cancer.

Table 2: Estradiol Levels By Quintiles of Prudent Pattern Score (23)

Estradiol (pg/mL)
Q1
Q2
Q3
Q4
Q5
P-value
$6.6(1.1)$
$7.4(1.1)$
$7.5(1.1)$
$6.7(1.1)$
$6.1(1.1)$
0.20

The numbers in the box indicate the estradiol levels of each quintile in $\mathrm{pg} / \mathrm{mL}$ for a prudent diet. Quintile 1 contains women who eat the least prudent diet and quintile 5 contain the women who eat the most prudent diet (23). The pvalue is listed to the right.

Table 3: Estradiol Levels By Quintiles of Western Score (23)

\section{Estradiol (pg/mL)}

$$
\text { Q1 }
$$

$$
\text { Q2 }
$$

Q3

Q4

Q5

P-value

Multivariate

$6.3(1.1)$

$6.6(1.1)$

$7.0(1.1)$

$7.1(1.1)$

$7.3(1.1)$

0.01

The numbers in the box indicate the estradiol levels of each quintile in $\mathrm{pg} / \mathrm{mL}$ for a western diet. Quintile 1 contains women who eat the least western diet and quintile 5 contains the women who eat the most western diet (23). The pvalue is listed to the right. 
Figure 4: Relative Risk of Breast Cancer in 238 Postmenopausal Women, According to the Serum Estradiol Concentration (24).

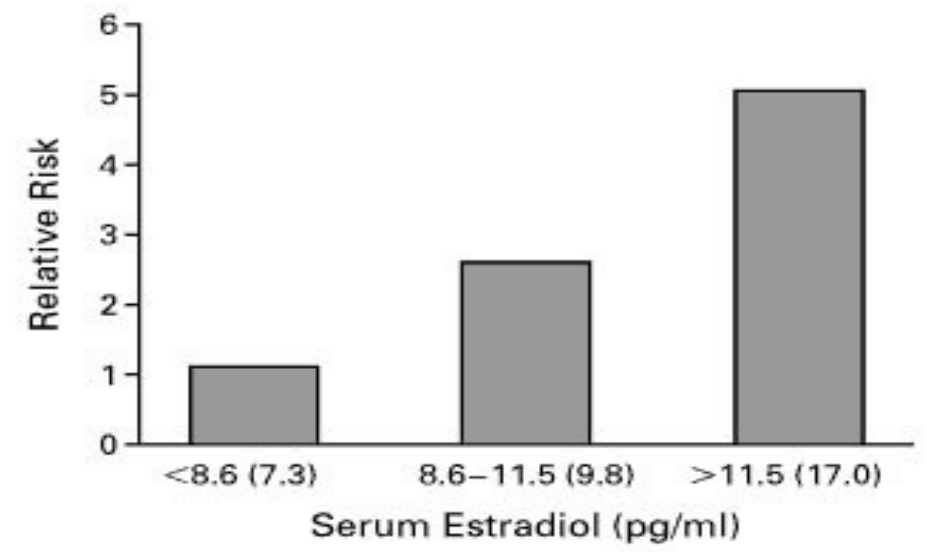

NO. OF WOMEN

With cancer

Without cancer

7
59

19

62
34

57

In this case-control study, 60 women with newly diagnosed cases of breast cancer and 178 controls participated in the study (43). The bar graph above shows the amount of serum estradiol (in $\mathrm{pg} / \mathrm{mL}$ ) in each group's bloodstream on the horizontal axis and the relative breast cancer risk on the vertical axis. For example, women with a serum estradiol of greater than $11.5 \mathrm{pg} / \mathrm{mL}$ in the bloodstream have a relative risk of scale 5 according to the graph (43).

\section{Discussion of Results}

This study's overall goal was directed towards finding a correlation between estrogenic diets and breast cancer risk. In the first table, Quartile 1 is labeled as the people who have the lowest estrogen pattern score and Quartile 5 is labeled as the people who have the highest estrogen pattern score (Table 1). The age-adjusted column was chosen since even though age is already known to be a factor of breast cancer, this table shows that the data was independent of age, therefore the data remains unaffected by that factor (Table 1). It shows that Quartile 1 has a hazard ratio of 1.00 ( $0 \%$ increased risk of breast cancer), Quartile 2, a hazard ratio of 1.10 (where it is a $10 \%$ increased risk of breast cancer), Quartile 3, a hazard ratio of 1.13 (where it is a 13\% increased risk of breast cancer), and Quartile 4, a hazard ratio of 1.30 (a 30\% increased risk of breast cancer) (Table 1). Since Quartile 5 has the highest estrogen diet pattern score, it is shown that they have an increase of $30 \%$ risk showing that there is a link between estrogenic diet and breast cancer risk (Table 1). In table 2, the prudent diet (which contains fruits, proteins, and veggies) were studied through estradiol levels. Quintile 1 which contains the people who eat the least prudent diet are shown to have estradiol levels of $6.6 \mathrm{pg} / \mathrm{mL}$, Quintile 2 with $7.4 \mathrm{pg} / \mathrm{mL}$, Quintile 3 with $7.5 \mathrm{pg} / \mathrm{mL}$, Quintile 4 with $6.7 \mathrm{pg} / \mathrm{mL}$, and Quintile 5 with $6.1 \mathrm{pg} / \mathrm{mL}$ (Table 2). This shows that there is no correlation between the estradiol levels and a prudent diet, since there is no clear increase or decrease in the data and as indicated by the p-value of .20, showing no statistical significance, since it is greater than .05 (Table 2). In table 3, the western diet (which contains foods with high estrogen, red meat, high sugar drinks, and fried foods) were studied through estradiol levels (Table 3). 
Quintile 1 which contains the people who eat the least western diet are shown to have $6.3 \mathrm{pg} / \mathrm{mL}$ of estradiol, Quintile 2 with $6.6 \mathrm{pg} / \mathrm{mL}$, Quintitle 3 with $7.0 \mathrm{pg} / \mathrm{mL}$, Quintile 4 with $7.1 \mathrm{pg} / \mathrm{mL}$, and Quintile 5 with $7.5 \mathrm{pg} / \mathrm{mL}$ (Table 3). This shows that there is a correlation between estradiol levels and a western diet, since there is a clear increase as indicated by the p-value (which is .01), a p-value that shows statistical significance. In figure 4, relative risk of breast and serum estradiol are graphed amongst each other. With serum estradiol levels less than $8.6 \mathrm{pg} / \mathrm{mL}$, the relative risk is 1 , while with levels greater than $11.5 \mathrm{pg} / \mathrm{mL}$, the relative risk is 5 showing that if estradiol levels are increased by about $3 \mathrm{pg} / \mathrm{mL}$, the risk of breast cancer increase by 5 times (Figure 4). Through these results, it is clear that western diets can increase estradiol levels, and estradiol levels can increase breast cancer risk significantly. It is also clear from the graph about prudent diets that the risk of breast cancer stays relatively constant, even after consumption (Table 2). In the first table, the relative risk of breast cancer tends to increase with an estrogen dietary pattern score (Table 1). Therefore, from these correlations, it is clear that foods containing high estrogen levels (western diets to be specific) increase risk of breast cancer significantly in women over age 50.

\section{Methods}

The first segment of the research was to explore more about what estrogen contains that causes breast cancer risk (for premenopausal and post-menopausal women), specifically from websites such as the National Cancer Institute, National Library of Medicine, and Susan G. Komen in order to ensure that the purpose of the study is viable (13, 22). I also researched more about the common foods containing estrogen on health websites (some examples include soy, edamame, red meat, western diet etc.) through health websites, published journals, and published blogs that are credible and have a medical background or reputation. In order to get a better idea on estrogen's relation to breast cancer and to also explore more on how diet can affect the risk and some common foods to avoid, I selected a book called "How Not to Die" by Michael Greger (25). I read approximately 10 pages of this per night for approximately 3 days as the section pertaining to breast cancer is not that lengthy. In order to answer my research question effectively, I first needed access to specific databases (GALE, Proquest), and health publishers like the National Cancer Institute, Susan G. Komen, National Library of Medicine, and International Journal of Cancer Research and Treatment. These sources were just a starting point, meaning that I needed to explore more sources since none of them addressed my topic extensively.

I first was interested in finding how breast cancer, estrogen, and diet relate to one another if at all. First, by searching the keywords breast cancer, estrogen, and dietary pattern score, the 2nd source that came up on Google Scholar (accessed through my expert advisor) was by the International Journal of Cancer Research and Treatment, a well-known global medical journal. I then found many tables that matched my topic from this source (diets with estrogenic properties and breast cancer), but one specific table titled "Relative risk of breast cancer by estrogen dietary pattern score among women" contained the exact information I was striving to include (22). To add more information to this data I obtained, I then searched up the keywords dietary patterns and plasma sex hormones and found a couple of sources by the National Library of Medicine at first. However, these were graduate school level articles, and they did not interpret the information in a clear manner. After browsing these sources, I then found a source by the International Journal of Cancer Research and Treatment explaining how prudent diets (veggies, fruits) and western diets (diets with estrogenic properties and red meats) affect breast cancer risk (23). Since I wanted to see if there was a link between western and prudent diets and estradiol levels in this research study, I decided to utilize that source. After finding this previous source, I then decided to search the keywords estradiol and risk of breast cancer, so I can therefore link the estradiol levels of western and prudent diets to breast cancer risk. I found a chart from the New England Journal of Medicine after browsing through a few medical journals explaining the relationship between estradiol levels and breast cancer risk (24). This served as a great figure to sum up my correlational research.

Before analyzing my collected data and finding correlations, a meeting with my expert advisor was scheduled about this analysis, so I can confirm that the data I collected was measuring what I thought it was. Since the data I utilized was online and no human subjects were involved, no face to face communication was required therefore 
increasing confidentiality (26). However, one anticipated challenge I partially faced included being too repetitive with studies, since many of the studies I decided to use all had similar objectives. My method intends to find how diets with high estrogen levels can increase breast cancer risk, so each study may have been very similar with minimal objective differences. My proposed research methods are ethical because it does not include any human subjects and is just researching about women's diets and how it is linked to breast cancer risk (27). Since I browsed through public databases, peer-reviewed journals, and health scholarly journals, the studies posted have been approved by the IRB Committee meaning they followed ethical processes (informed consent, debriefing, etc) (27). I also made sure I cited each source and gave credits to all the authors for these sources as well in order to demonstrate credibility of the information.

Next, after obtaining the sources I described above, I analyzed if foods that the women eat on a daily basis correlate with the levels of estradiol and thus analyze if that estradiol level increases or decreases their risk of breast cancer. However, each participant had to be above 50 to be counted in my study. I then looked through the studies and first checked if there was a correlation between diet and their estrogen levels and next, between estrogen levels and breast cancer to determine if the particular diets high in estrogen increase breast cancer risk. To make sure the data is viable, I recorded the findings discussed in the meetings with my expert advisor to ensure that the correlation was accurate and precise. To be fit inside the research paper, the data was organized in tables for each research study (totaled up to 300 participants) which was included in my research paper. The setting used in all of the sources I obtained data from were all closed settings, therefore increasing the statistical significance of the results (28). I then recorded my findings in the discussion section of the academic paper which included a proofread from my expert advisor to ensure validity.

\section{Acknowledgments}

I would like to thank my advisor Dr. Aubrey Frantz from the University of North Texas for her valuable insight and help with this project.

\section{References}

1. Cancer Facts for Women. American Cancer Society. https://www.cancer.org/healthy/find-cancer-early/womens-health/cancer-facts-for-women.html. Updated July 30, 2020. Accessed October 10, 2020.

2. Cancer Statistics. National Cancer Institute. https://www.cancer.gov/aboutcancer/understanding/statistics\#: :text=Approximately $\% 2039.5 \% 25 \% 20 \mathrm{of} \%$ 20men $\% 20$ and,will\%20die \%20of\%20the\%20disease. Updated September 25, 2020. Accessed October 10, 2020.

3. Hollern D., Swiatnicki M., Andrechek E. "Can Mice Really Mirror Humans When it Comes to Cancer?" Science Daily. vol. 14, no. 1, 2018, pp. 178-180; DOI: e1007135

4. Tiberian J.. "4 Tips to Help Prevent Estrogen Dominance, A Risk for Breast Cancer in Women and Men." MDVIP. https://www.mdvip.com/about-mdvip/blog/understanding-estrogen-dominance-risk-breast-cancer-womena nd-men Published 2018 November 15. Accessed 2020 October 10.

5. Kotepui M. "Diet and Risk of Breast Cancer”. US National Library of Medicine. Vol. 20, no. 1, $2016, \mathrm{pp}$. 13-19; DOI: 10.5114/wo.2014.40560

6. Harding, C., Pompei, F., et al. "Cancer Suppression at Old Age." American Association for Cancer Research Journals. 2008; DOI: 10.1158/0008-5472.CAN-07-1670 
7. Zeng, X., Douglas Y.. "Insulin-Like Growth Factors and Breast Cancer Therapy.” US National Library of Medicine. 2000-2013; Madame Curie Bioscience Database [Internet].

8. Can I Lower My Risk of Breast Cancer Progressing or Coming Back? American Cancer Society. https://www.cancer.org/cancer/breast-cancer/living-as-a-breast-cancer-survivor/can-i-lower-my-risk-ofbrea st-cancer-progressing-or-coming-back.html. Updated June 9, 2020. Accessed October 17, 2020.

9. Patisaul, H., Jefferson W.. "The pros and cons of phytoestrogens." US National Library of Medicine. Vol. 31, no. 4, 2011, pp. 400-419; DOI: 10.1016/j.yfrne.2010.03.003;

10. Treatments for Hormone-Receptor-Positive Breast Cancer. Breast Cancer Research News. https:/www.breastcancer.org/symptoms/diagnosis/hormone_status/treatment_hrpos. Updated September 21, 2020. Accessed October 17, 2020.

11. Watkins, Elyse. "Overview of Breast Cancer.” Journal of the American Academy of Physician Assistants. Vol. 32, no. 10, 2019, pp. 13-17

12. Darbre, Philippa., Charles A.. "Environmental Oestrogens and Breast Cancer: Evidence for Combined Involvement of Dietary, Household and Cosmetic Xenoestrogens." International Journal of Cancer Research and Treatment. Vol. 30, no. 3, 2010, pp. 815-827

13. Wu, Anna., Pike, Malcolm.. "Meta-analysis: Dietary Fat Intake, Serum Estrogen Levels, and the Risk of Breast Cancer.” Journal of the National Cancer Institute. Vol. 91, no. 6, 1999, pp. 529-534, DOI: https://doi.org/10.1093/jnci/91.6.529;

14. Norton, Amy.. "Estrogen-like lignan diet, less breast cancer linked." American Journal of Clinical Nutrition. https://www.reuters.com/article/us-estrogen/estrogen-like-lignan-diet-less-breast-cancer-linkedidUSTRE6 535S220100604 Published 2010 June 4. Accessed 2020 November 21.

15. Caffa, Irene., Spagnolo, Vanessa. "Fasting diet could boost breast cancer therapy." Science Daily. Vol. 583, no. 7817, 2020, pp. 533-581, DOI: 10.1038/s41586-020-2502-7

16. Ledesma, Natalie. "Nutrition \& Breast Cancer." UCSF Medical Center. https://cancer.ucsf.edu/_docs/crc/nutrition_breast.pdf Published 2013 June 28. Accessed 2020 November 21.

17. Caffa, Irene., Spagnolo, Vanessa.. "Fasting-mimicking diet and hormone therapy induce breast cancer regression." Nature. Vol. 583, no. 1, 2020, pp. 620-624

18. Science.gov.. "Sample records for experimental research designs." Science.gov. https://www.science.gov/topicpages/e/experimental+research+designs.html Published 2011 Janurary 18. Accessed 2020 November 21

19. Survey and Correlational Research Designs? SagePub. https:/www.sagepub.com/sites/default/files/upm-binaries/57732_Chapter_8.pdf Updated February 9, 2020. Accessed 2020 November 20

20. Xiaohui, Liang. "The Menstrual Cycle Related Hormone Variations and Breast Cancer Risk: A Novel Theory." Science Repository. Vol. 1, no. 1, 2019, pp. 133-139, DOI: 10.31487/j.COR.2020.04.01

21. Lu, Jane., Anderson, Karl..”Decreased Ovarian Hormones during a Soya Diet: Implications for Breast Cancer Prevention." American Association for Cancer Research. Vol. 60, no. 15, 2000, pp. $4112-$ 4121

22. Harris, Holly., Bergkvist, Leif.. "An estrogen-associated dietary pattern and breast cancer risk in the Swedish Mammography Cohort.” PubMed. https://pubmed.ncbi.nlm.nih.gov/25924604/\#: :text=A\%20higher\%20estrogen\%20dietary\%20pattern,(tre n d)\%20\%3D\%200.006. Published 2015 November 1. Accessed February 22.

23. Clemons, Mark., Goss, Paul. "Estrogen and the Risk of Breast Cancer." The New England Journal of Medicine. https://www.nejm.org/doi/full/10.1056/nejm200101253440407. Published 2001 January 25. Accessed March 28. 
24. Friedenreich, Christine., MacLaughlin, Sarah. "Study design and methods for the Breast Cancer and Exercise Trial in Alberta (BETA)". US National Library of Medicine. vol. 919, no. 19, 2014, pp. 333359. DOI: $10.1186 / 1471-2407-14-919$

25. Eating Soy May Turn on Genes Linked to Cancer Growth. BreastCancer.Org https://www.breastcancer.org/research-news/soy-may-turn-on-genes-linked-to-cancer. Updated September 5, 2014. Accessed November 17, 2020.

26. Tonkelaar, I. den., Waard, F. de. "Regularity and length of menstrual cycles in women aged 41-46 in relation to breast cancer risk: Results from the DOM-project." SpringerLink., vol. 38; 1996, pp. 253258; DOI: 10.1007/BF01806143

27. How Not to Die, an instant New York Times Bestseller. NutritionFacts.org. https://nutritionfacts.org/book/how-not-to-die/ Updated 2020 August 28. Accessed 2020 December

28. Marchione, Marilynn. "Less fat, more fruit may cut risk of dying of breast cancer." APNews. https://apnews.com/article/b8ecabb3303f4243a8b353f1adadc163 Published 2019 May 15. Accessed 2020 November 22.

29. Chapter 10 Correlational Research. Lumen Learning. https://courses.lumenlearning.com/suny-hccc-research-methods/chapter/chapter-10-experimentalresearch/ Updated April 9 2012. Accessed November 232020.

30. Fitzgerald, Emily. “A New Link Between Diet and Breast Cancer Treatment. What does it mean?" Ottawa Regional Cancer Foundation. https://www.ottawacancer.ca/diet-and-breast-cancer-treatment/ Published 2019 May 22. Accessed 2020 November 23

31. Hensley, Martee. “Can Eating Soy Affect Breast Cancer Recurrence?” Everyday Health. https://www.everydayhealth.com/breast-cancer/managing/hensley/soy-affect-breast-cancerrecurrence.aspx Published 2008 June 9. Accessed 2020 November 23

32. Calado, Ana., Neves, Pedro. "The Effect of Flaxseed in Breast Cancer: A Literature Review." Frontiers in Nutrition. https://www.frontiersin.org/articles/10.3389/fnut.2018.00004/full Published 2018 February 7. Accessed 2020 November 23

33. Fruits, Vegetables, \& Carotenoids. Susan G. Komen. https://www.komen.org/breast-cancer/risk-factor/lifestyle/fruits-vegetables-carotenoids/ Updated 2019 November 18. Accessed 2020 December 9

34. How to Create Powerful Online Surveys that Get Completed. DragonSurvey. https:/www.dragnsurvey.com/blog/en/how-to-create-powerful-online-surveys-that-get-completed/ Updated 2010 June 28. Accessed 2020 December 9

35. How does Menopause affect Cancer Risk? The University of Texas MD Cancer Center. https://www.mdanderson.org/publications/focused-on-health/FOH-menopause-cancer.h201589835.html. Updated 2015 November. Accessed 2020 December 10

36. Mastalerz, Ania. "DIY Recruiting: How to Find Participants for Your Research." Mixed Methods. https://medium.com/mixed-methods/diy-recruiting-how-to-find-participants-for-your-research6f9a05dd1a 33. Updated 2017 November 21. Accessed 2020 December 10

37. Suzuki, Reiko., Ye, Weimin.. "Dietary fiber intake and risk of postmenopausal breast cancer defined by estrogen and progesterone receptor status--a prospective cohort study among Swedish women." National Library of Medicine. https://pubmed.ncbi.nlm.nih.gov/17764112/. Published 2008 January 15. Accessed February 22.

38. Larsson, Susanna., Bergkvist, Leif.. "Long-term meat intake and risk of breast cancer by oestrogen and progesterone receptor status in a cohort of Swedish women." National Library of Medicine. https://pubmed.ncbi.nlm.nih.gov/19464165/. Published 2009 November. Accessed February 22.

39. Fung, Teresa., Hu, Frank.. "Diet Quality Is Associated with the Risk of Estrogen Receptor-Negative Breast Cancer in Postmenopausal Women." The Journal of Nutrition. 
https://academic.oup.com/jn/article/136/2/466/4743764?login=true--. Published 2006 February 1. Accessed February 22.

40. Heath, Alicia., Muller, David. "Nutrient-wide association study of 92 foods and nutrients and breast cancer risk." Biomedical Central.

https://breast-cancer-research.biomedcentral.com/articles/10.1186/s13058-019-1244-7. Published 2020 January 13. Accessed February 22.

41. Fung, Teresa., Hu, Frank., Barbieri, Robert, et. al. "Dietary patterns, the Alternate Healthy Eating Index and plasma sex hormone concentrations in postmenopausal women." International Journal of Cancer. https://onlinelibrary.wiley.com/doi/full/10.1002/ijc.22728. Published 2007. Accessed March 28.

43. Sauter, Edward. "Breast Cancer Prevention: Current Approaches and Future Directions.” US National Library of Medicine. https://www.ncbi.nlm.nih.gov/pmc/articles/PMC5939980/. Published 2018 April 1. Accessed April 12.

44. Costa, Mauricio., Saldanha, Paula. "Risk Reduction Strategies in Breast Cancer Prevention.” US National Library of Medicine. https://www.ncbi.nlm.nih.gov/pmc/articles/PMC5544140/. Published 2017 July 1. Accessed April 12. 